The onset of shear modes in the high frequency spectrum of simple disordered systems: current knowledge and perspectives

Alessandro Cunsolo ${ }^{\S}$, Alexey Suvorov and Yong Q. Cai

Photon Sciences Directorate-Brookhaven National Laboratory, P.O. Box 5000 Upton, NY, USA.

${ }^{\S)}$ Corresponding author: acunsolo@bnl.gov

Tel. 0016313445564

Fax 0016313448189 


\title{
The onset of shear modes in the high frequency spectrum of simple disordered systems: current knowledge and perspectives
}

\begin{abstract}
Nearly two decades of thorough Inelastic X Ray Scattering (IXS) studies of transverse-like excitation in the spectrum simple amorphous materials are reviewed. A particular attention is given to the case of liquid water and other prototypical samples, through a discussion of both solved and still open issues. Finally, the perspectives opened up by the development of next generation IXS instruments with unprecedented contrast and resolution bandwidth are briefly illustrated.
\end{abstract}

Keywords: inelastic X ray scattering; non-hydrodynamic modes; disordered systems

\section{Introduction}

In the last decades the search for non-hydrodynamic, non-longitudinal collective modes in the spectrum of density fluctuations, $S(Q, \omega)$, of liquids has received a growing attention. Although the onset of shear modes in $S(Q, \omega)$ has been amongst the most thoroughly studied topics in this field, it still presents some controversial aspects which makes it worth further investigations.

Historically, the presence of shear mode in the $\mathrm{S}(\mathrm{Q}, \omega)$ has been first observed in liquid water [1-5] by inelastic spectroscopy, however it could be unambiguously assessed only with the support of molecular dynamics (MD) simulation. In fact MD methods can selectively determine transverse and longitudinal current spectra, respectively defined as Fourier transform of the correlation function between either longitudinal or transverse components of center of mass molecular velocities. Although the synergy of spectroscopic and computational results represents a powerful method to pinpoint the onset of shear modes in the $\mathrm{S}(\mathrm{Q}, \omega)$, this topic has often raised some skepticism especially for liquid water [6,7].

In principle, the onset of a transverse mode in the spectrum of a disorder material should not be surprising; in fact quasi-polarized (transverse or longitudinal) modes are known to contribute to the inelastic spectra of poly-crystals in the first-Brillouin zone, while, in a crystal, a pure polarization of a mode can only be observed along high symmetry directions, which are not relevant for a liquid. Therefore the assumption that in a liquid transverse and longitudinal polarization may be intertwined may is not ungrounded, especially over distances matching the size of molecular disorder.

Still, it is broadly accepted that a longitudinal or transverse polarization can be assigned to a mode propagating through a liquid at a mesoscopic wavelength, if such a mode dominates the current spectra of corresponding -longitudinal or transverse- polarization. A possible objection follows from the circumstance that $S(Q, \omega)$ primarily couples with longitudinal movements only, which would suggest to exclude the presence of a pure transverse mode in the $\mathrm{S}(\mathrm{Q}, \omega)$. Indeed, in a liquid, the coupling of density fluctuations with shear waves, can only occur indirectly via the so called L-T mixing $[2,8,9]$, that is a coupling between modes of longitudinal and transverse polarization. This coupling is active only above some Q-threshold, $\mathrm{Q}_{\mathrm{t}}$, which, for instance, in water was found to be either 4 or $\approx 6 \mathrm{~nm}^{-1}$ according to Ref. [1] and [4] respectively.

In spite of the merely qualitative nature of the above arguments, the body of available literature results in the field outlines a rather coherent scenario: owing to the lack of translational invariance typical of liquids, longitudinal and transverse modes become 
mutually intertwined and their symmetry somehow ill-defined over distances shorter than some threshold value $\lambda=\mathrm{Q}_{\mathrm{t}}^{-1}$. This distance, in principle dependent upon both sample characteristics and thermodynamic conditions, marks the viscoelastic transition from a liquid-like (viscous) and a solid-like (elastic) regime of the dynamic response of the fluid. Only at wavelengths shorter than $\lambda$ the response of system has reached its "elastic" regime and a transverse mode become in principle observable in the spectrum of density fluctuations.

Consistently with this scenario, an IXS work on liquid water covering a broad thermodynamic range [2] also demonstrated that an L-T coupling only occurs when density fluctuations propagate at frequencies larger than the inverse of the relaxation time $1 / \tau$. Under these conditions, these acoustic waves "perceive" the propagation medium as frozen, or solid-like, as a glass. Its internal degrees of freedom become thus too slow to efficiently couple with the acoustic wave, which therefore travels "elastically", that is keeping its energy constant. The virtual lack of acoustic dissipation typical of this regime causes a higher sound velocity and the lower sound damping. Furthermore, it causes restoring forces to more effectively support a sound propagation in the shear plane. Although there is a large body of literature supporting the soundness of the above scenario, several unsolved topics are still associated to L-T phenomenon and they mainly concern the microscopic mechanism activating it and a possible universal link with the interatomic potential and/or first neighbor arrangement of the fluid. At present the microscopic explanation of this phenomenon seems still elusive, since, as discussed in following paragraphs, a L-T mixing has been observed in fluids with very disparate intermolecular interactions and thermodynamic conditions, as well as in more complex systems, such as mixtures [10] and biophysical samples [11-13], which, however, are not discussed in the present work.

The aim of this short review is to provide a concise account of the advances in this field achieved across the years, with particular emphasis to the case of liquid water and to the perspectives opened by the development of novel IXS spectrometers with improved spectral contrast and energy resolution.

\section{The case of liquid water}

Among various liquid systems whose $\mathrm{THz}$ spectrum bears evidence of shear mode propagation, water is definitely the most thoroughly studied. Relevant experimental studies on this sample date back to the seminal Raman work of Segre' [14] and the three successive works of Bolla [15-17], showing that the low frequency portion of the Raman spectrum of water is dominated by two modes at nearly $7.4 \mathrm{meV}$ and $22 \mathrm{meV}$. The collective character of both low and high frequency modes was clear since then, although the respective transverse and longitudinal polarization was assigned only much later. This assignment is one of the main otcomes of more recent Raman studies [18-20] connecting these modes to the propagation of acoustic waves hindered by the existing hydrogen bond (HB) network. In particular, according this explanation shear acoustic waves in water are hampered by the bending of three $\mathrm{O}$ atoms belonging to distinct HBlinked molecules, while longitudinal waves mainly interfere with the O-O HB stretching. Equivalently, these acoustic modes could be identified as the counterparts in liquid water of the transverse (TA) and the longitudinal (LA) acoustic mode observed in the spectrum of hexagonal ice [21,22]. In this respect it is worth mentioning that the intermolecular bending mechanism in ice has a mere transverse character [21]. 
However, no evidence was reported on the presence of these modes in the $S(Q, \omega)$ of liquid water, until the pioneering Molecular Dynamics (MD) work of Rahman and Stillinger [23]. Among various results, this computational study demonstrated that at $\mathrm{THz}$ spectrum of water was dominated by two inelastic modes. The lower frequency one was identified as the finite-Q extension of the hydrodynamic sound mode and thereafter often quoted as "normal sound". The high frequency mode, thereafter referred to as "fast sound", propagated with a velocity resembling the speed of longitudinal phonon of ice and was later assumed to propagate throughout ice-like patches of liquid water [24].

Although back then popular, nowadays the nomenclature originally adopted for the two modes seems misleading or, at best, obsolete [25-27], however it is here maintained for historical purposes only. The presence of a "normal sound" mode was confirmed by two Inelastic Neutron Scattering (INS) measurements [28,29]. and a MD simulation [30], which also proposed a similar explanation of its link with the hydrodynamic sound mode. However, the latter interpretation seemed somehow in conflict with previous INS measurements on neon [31] rubidium [32] and lead [33], which indicated a steep Qincrease of the sound dispersion beyond the hydrodynamic limit, rather than the flat Qdependence found for the "normal sound" mode. A detailed account of the controversy around the actual existence of a "fast sound" mode in water goes beyond the present scope and can be found, for instance, in Refs. [25] and [26].

From the experimental side, kinematic limitations [34] hampering the only $\mathrm{THz}$ spectroscopic technique available back at those times, INS, could be overcome only after the development of the first high resolution IXS in the mid-nineties ID16 beamline at ESRF [35,36]. In particular the three IXS measurements performed in the early operation phase this instrument [1,5,37] slightly extended the spanned Q region and substantially broadened the covered frequency range.

As a result, it was confirmed the double-peaked structure of water spectrum previously observed by Rahman and Stillinger [23]. However, the low frequency peak could only be detected for $\mathrm{Q}$ larger than $4 \mathrm{~nm}^{-1}$, which led to exclude the hypothesized low $\mathrm{Q}$ evolution toward the hydrodynamic sound mode. Indeed, the authors ascribed this low frequency peak the onset of a shear propagation based upon a comparison with the case of ice where a peak was observed at similar frequency [5] and previously assigned to a transverse optical mode [22]. Nearly contemporary MD studies proposed a similar interpretation of a low frequency peak in the velocity autocorrelation function of water [38]. Furthermore, the presence of a transverse mode was clearly identified in the MD computed longitudinal current spectrum of water and the investigation of its $Q$ dependence represents the first compelling evidence for an L-T coupling in water [9].

A deeper insight on the phenomenology of the L-T coupling and its link with the viscoelastic properties of water can be gained from the comparison of MD [8] and IXS results [2], whose main outcome is summarized by Figure 1. The left plot clearly shows that the computed transverse and longitudinal current spectra of water are dominated by the same two inelastic modes. Furthermore, their dispersion curves essentially coincide with the ones of the low and high frequency peaks appearing the IXS spectrum (see right plot). The existence of common peaks in the longitudinal and current spectra demonstrated the intertwined nature of longitudinal and transverse modes at mesoscopic scales in water, or, equivalently, the occurrence of an L-T coupling.

As mentioned in the introductory section, IXS results discussed in Ref. [2] demonstrated that the onset of a transverse mode in the water spectrum has a precursor in the viscoelastic transition of the longitudinal sound velocity induced by a structural relaxation [39-41]. 
In the work of Cimatoribus and collaborators [3] it was also suggested that, at low temperatures, the interaction of the $\mathrm{THz}$ spectrum with shear waves is definitely more complex than suggested by previous experimental and computational results. In fact, in (or close to) supercooling conditions a further weakly dispersing high frequency mode becomes clearly visible in the inelastic wings of the spectrum.

Finally, it is worth mentioning a joint IXS and INS study on deuterated water [4], which combined the superior resolution and spectral contrast provided by INS with the large dynamic range covered by IXS. This synergy has permitted a proper simultaneous detection of both the low frequency (transverse) and high frequency (longitudinal) mode in the spectrum, even at ambient temperature. A comparison between the two parallel measurements performed in Ref. [4] is proposed by Figure 2: the left panel compares the $S(Q, \omega)$ of $D_{2} \mathrm{O}$ measured by the two complementary techniques, while the right panel show the current spectra derived from them through $C_{L}(Q, \omega)=(\omega / Q)^{2} S(Q, \omega)$. The comparison between spectral profiles evidences the complementarity of the measurements. The much narrower $(<.1 \mathrm{meV})$ and sharper - essentially Gaussian - INS resolution is essential for a proper detection of the low frequency peak. On the other hand, the virtual absence of kinematic limitations allows the IXS measurement to cover the region of the longitudinal mode.

Among various results, Ref. [4] demonstrated that the crossover from the viscoelastic regime toward the elastic one is accompanied by the corresponding increase of the relative transverse damping $\mathrm{R}_{\mathrm{t}}=\Gamma_{\mathrm{t}} / \Omega_{\mathrm{t}}$ with $\Gamma_{\mathrm{t}}$ and $\Omega_{\mathrm{t}}$ being the width and the inelastic shift of the transverse peak, respectively. In the viscoelastic regime $\left(\Omega_{t} \tau \approx 1\right)$ the transverse mode has the form of an overdamped $\left(R_{t} \leq 1\right)$ shoulder, essentially merging into the Mountain [42] relaxation peak. Conversely at higher $\mathrm{Q}$ values it becomes increasingly underdamped $\left(\mathrm{R}_{\mathrm{t}} \geq 1\right)$ gradually transforming into a relatively long-lived inelastic peak.

\section{The onset of a transverse dynamics in other systems}

The presence of transverse modes in the spectrum of glass-forming or glassy materials has often been connected to the low frequency excess in the density of states, DOS. This is known to give rise to a peak in the reduced density of states DOS/ $\mathrm{E}^{2}$, customarily referred to as the Boson peak. More recently, it has been demonstrated $[43,44]$ that the $\mathrm{BP}$ can be considered as the equivalent of the transverse acoustic (TA) van Hove singularity of crystals, shifted to lower energy by the force-constants disorder. It is worth recalling that van Hove singularities are poles in the density of states located at the boundary of the first Brillouin zone, where $\partial \omega_{\mathrm{s}} / \partial \mathrm{Q}=0$ (with $\omega_{\mathrm{s}}$ being the sound dispersion curve). This condition is certainly satisfied by transverse modes in most liquids which seem to exhibit nearly flat Q-dependence at intermediate Qs.

Interestingly, some MD simulation results on silica did not fit into this scenario [45] indicating that transverse current spectra are dominated by a low frequency modes linearly dispersing up to energies much higher than those characteristic of the BP. In Ref. [45] it was also observed that the TA peak of silica has an amplitude 6-8 times larger than the longitudinal acoustic (LA) one. This is most likely due to the stiffness of the covalent tetrahedral network, which introduces strong restoring forces fostering the propagation of large-amplitude shear waves even at extremely high temperatures (> $3000 \mathrm{~K})$. Transverse modes in the spectrum of $\mathrm{SiO}_{2}$ are likely interacting to hindered librations of $\mathrm{SiO}_{4}$ tetrahedra [46], in some analogy with the previously discussed case of water in which they are assumed to interact with the bending of HB-linked O-O-O triplets [20]. It was also suggested that the visibility of the transverse mode in the 
spectrum of density fluctuations was made possible by a change in polarization caused by tetrahedra rotations [47].

Similar L-T coupling effects were experimentally observed in several tetrahedral systems. Beside of the discussed cases of water and silica, those of amorphous $\mathrm{GeO}_{2}$ [48] and $\mathrm{GeSe}_{2}$ [49], also deserve a mention. This seems to support the assumption that the L-T coupling phenomenon is somehow connected to the tetrahedral geometry of bonds arrangements. In fact the intrinsically open (large free volume) and directional character of this geometry is often expected to favour a coupling between orthogonal (longitudinal and transverse) movements of the liquid's molecules.

The occurrence of a L-T coupling has been computationally observed in other associated systems, such as glassy glycerol [50]. Interestingly, the analysis of the temperature dependence of the L-T coupling in this material led to exclude its possible intramolecular origin, being likely connected to the HB network.

In this respect it may be surprising that an analogous L-T coupling was observed also in more closely packed and non-associated materials as liquid Ga [51-53]. Indeed, this systems appears to be a rather peculiar as a liquid metal since it has been shown [54] that the coexistence of metallic and covalent bonds in it gives rise to very short-lived Ga-Ga bonds, likely reminiscent of crystalline form $\alpha-G a$. In this respect it's not surprising that a solid-like feature such as the onset of a transverse mode in the $S(Q, \omega)$ is reported by several IXS works on this sample.

The assignment of a transverse origin to measured low frequency mode in the spectrum of gallium either stemmed from a comparison with measurements on the crystal [53] or with $a b$ initio MD calculations [51,52]. In particular, it was found that this mode closely resembles the TA mode of the $\beta$-Ga crystalline phase, yet with a substantially larger damping dominated by both elastic anisotropy and structural disorder [53]. In Ref. [52] it was also hypothesized that the ability of liquid $\mathrm{Ga}$ in supporting a transverse mode is fostered by solid-like cage effect on the nanometer scale acting as a restoring force for the shear density waves. This is also likely connected to the anomalously high value estimated for the Poisson ratio (0.42), which turns out to be comparable to the one of rubber-like materials.

An IXS work on sodium showed the presence in the spectrum of both longitudinal and transverse acoustic excitations at frequencies strictly related to the corresponding ones in the crystal phase [55]. This works offeed an interesting perspective, since it suggested that the disorder-induced contribution to the acoustic damping in liquid is closely related to the distribution of local structures around the average one. From this argument one can qualitatively expect that, at low enough temperatures, such a distribution can become so sharp to make the shear fluctuation underdamped $\left(R_{t}<1\right)$, that is cause transverse modes to propagate. This clearly suggest a link between dynamical and structural effects which can be potentially useful for a deeper understanding of the L-T phenomenon.

A study of transverse mode in Sn [56] suggested that inelastic modes in this metal are strongly localized being their propagation length $\pi / \mathrm{QR}_{\mathrm{t}}$ smaller than the Ioffe-Riegel distance (see, e.g., Ref. [57]). It is finally worth mentioning a very recent work in liquid Zn [58], in which a second excitation with a transverse acoustic-like nature was observed and ascribed to peculiar anisotropic interactions of this system.

\section{Future perspective: the contribution of next generation IXS instruments}

As clear from Fig. 2 a proper detection of the low frequency transverse peak the THz spectrum of water requires an extremely sharp and narrow (sub-meV) resolution, 
currently attainable with INS instruments only. On the other hand, IXS offers unique and complementary advantages as the access to low Qs and the virtually unlimited kinematic range covered, which are key factors for a complete characterization of the L$\mathrm{T}$ coupling. This can explain the critical need for new generation inelastic spectrometer combining the best complementary assets of the two THZ spectroscopic techniques.

On a general ground, a more firm understanding of the spectral shape at both low and high frequencies is crucial for any advance in this field. In fact shear relaxations leading to shear mode propagation are expected to have signature in both these opposite frequency regions. This cannot be achieved without a substantial improvement of current spectrometers' performance: a high contrast and a large dynamic range are essential to reliably measure the extreme spectral wings, while a high contrast and a narrow resolution are required to determine its low frequency portion.

A great hope is held by forthcoming new generation IXS spectrometer conjugating the best characteristics of state of art IXS and INS techniques.

Although not yet ready for the use of scientific community, one of such spectrometers is soon becoming available at the IXS beamline of the new synchrotron source NSLS-II at Brookhaven National Laboratory [59]. A schematic layout of such a beamline is proposed in Fig. 3.

The energy scans will be implemented using the angular dispersion of asymmetrically cut crystals without any of differential temperature scanning [60]. This represents a substantial simplification since the achievement of a meV broad resolution bandwidth in state-of-art IXS instrument imposes a temperature control/stability better than $10^{-4} \mathrm{~K}$ for all active X-ray optics. The monochromator uses an optical scheme consisting in an array of four Si crystals customarily referred to as 4-bounce array [61], while the energy analysis is implemented by an optical setup consisting in a collimator (C) a dispersive (D) and a wavelength selector (W) crystal and referred to as CDW [62].

This combination demonstrated to provide viable routes to the achievement of a submeV broad and extremely sharp resolution profiles. Furthermore these optical schemes provide a wealth of advantages as compared to current backscattering IXS optics:

1) The access to high order Bragg reflection is not required in these schemes, which permits to circumvent severe intensity penalties.

2) The spectral contrast, i.e. the sharpness of the resolution function is dramatically enhanced by the multiple bunches in both monochromator and analyser units.

3) These assemblies are ideally operated at moderate energies at which many current synchrotron undulators perform best.

4) They offer a higher flexibility, including the opportunity of selecting nearly any energy and the capability of tailoring the resolution to a specific energy value.

The working principle of one of such schemes has been demonstrated in a recent work [63], which, among other results, presented the first sub-meV resolution IXS measurement of the glycerol spectrum (see Fig. 4). Owing to the pioneering character of such a measurement, the statistical accuracy in the spectral acquisition is still far from the standards of current IXS instrumentation. However, the improvement in the spectral definition strikingly emerges from the comparison between the broad conventional IXS profile and the sharp triplet line-shape captured by the new generation IXS measurement. This result is certainly quite encouraging and, in the foreseeable future, deemed to further advance our knowledge of the complex THZ dynamics of disordered materials. 


\section{References}

[1] F. Sette, G. Ruocco, M. Krisch, C. Masciovecchio, R. Verbeni, U. Bergmann, Phys. Rev. Lett. 77 (1996) 83.

[2] E. Pontecorvo, M. Krisch, A. Cunsolo, G. Monaco, A. Mermet, R. Verbeni, F. Sette, G. Ruocco, Phys. Rev. E 71 (2005) 011501.

[3] A. Cimatoribus, S. Saccani, F. Bencivenga, A. Gessini, M.G. Izzo, C. Masciovecchio, N. J. Phys. 12 (2010).

[4] A. Cunsolo, C.N. Kodituwakku, F. Bencivenga, M. Frontzek, B.M. Leu, A.H. Said, Phys. Rev. B 85 (2012).

[5] G. Ruocco, F. Sette, U. Bergmann, M. Krisch, C. Masciovecchio, V. Mazzacurati, G. Signorelli, R. Verbeni, Nature 379 (1996) 521.

[6] C. Petrillo, F. Sacchetti, B. Dorner, J.B. Suck, Phys. Rev. E 62 (2000) 3611.

[7] F. Sacchetti, J.B. Suck, C. Petrillo, B. Dorner, Phys. Rev. E 69 (2004) 061203.

[8] M. Sampoli, G. Ruocco, F. Sette, Phys. Rev. Lett. 79 (1997) 1678.

[9] U. Balucani, J.P. Brodholt, R. Vallauri, Journal of Physics: Condensed Matter 8 (1996) 9269.

[10] A. Cunsolo, C.N. Kodituwakku, F. Bencivenga, A.H. Said, The Journal of chemical physics 139 (2013) 184507.

[11] A. Paciaroni, A. Orecchini, M. Haertlein, M. Moulin, V. Conti Nibali, A. De Francesco, C. Petrillo, F. Sacchetti, The journal of physical chemistry. B 116 (2012) 3861.

[12] N. Violini, A. Orecchini, A. Paciaroni, C. Petrillo, F. Sacchetti, Phys Rev B 85 (2012).

[13] M.D. Li, X.Q. Chu, E. Fratini, P. Baglioni, A. Alatas, E.E. Alp, S.H. Chen, Soft Matter 7 (2011) 9848.

[14] E. Segre', Rend. Lincei 18 (1931) 929.

[15] G. Bolla, Nuovo Cimento D 9 (1932) 290.

[16] G. Bolla, Nuovo Cimento D 10 (1933) 101.

[17] G. Bolla, Nuovo Cimento D 12 (1935) 243.

[18] G.E. Walrafen, J. Chem Phys. 40 (1964) 3249.

[19] G.E. Walrafen, J. Chem Phys. 44 (1966) 1546.

[20] G.E. Walrafen, Y.C. Chu, G.J. Piermarini, J Phys Chem 100 (1996) 10363.

[21] J.E. Bertie, The Journal of chemical physics 46 (1967) 1271.

[22] B. Renker, Phys Lett A A 30 (1969) 493.

[23] A. Rahman, F. Stillinger, Phys. Rev. A 10 (1974) 368.

[24] J. Teixeira, M.C. Bellissent-Funel, S.H. Chen, B. Dorner, Phys. Rev. Lett. 54 (1985) 2681.

[25] G. Ruocco, F. Sette, Condens. Matter Phys. 11 (2008) 29.

[26] A. Cunsolo, Advances in Condensed Matter Physics 2015 (2015) 1.

[27] S. Santucci, D. Fioretto, L. Comez, A. Gessini, C. Masciovecchio, Phys. Rev. Lett. 97 (2006).

[28] P. Bosi, F. Dupre, F. Menzinger, F. Sacchetti, M.C. Spinelli, Lett. Nuovo Cimento 21 (1978) 436.

[29] F.J. Bermejo, M. Alvarez, S.M. Bennington, R. Vallauri, Phys. Rev. E 51 (1995) 2250.

[30] M.A. Ricci, D. Rocca, G. Ruocco, R. Vallauri, Phys. Rev. Lett. 61 (1988) 1958.

[31] H. Bell, H. Moellerwenghoffer, A. Kollmar, R. Stockmeyer, T. Springer, H. Stiller, Phys. Rev. A 11 (1975) 316.

[32] J.R.D. Copley, J.M. Rowe, Phys. Rev. Lett. 32 (1974) 49.

[33] O. Soderstrom, J.R.D. Copley, J.B. Suck, B. Dorner, J Phys F 10 (1980) L151. 
[34] S.W. Lovesey, Theory of neutron scattering from condensed matter, Clarendon Press, Oxford, 1984.

[35] M. Krisch, F. Sette, Neutron and X Ray Spectroscopy, Springer Verlag, Berlin, 2007, p. 317.

[36] G. Ruocco, F. Sette, Condens. Matter Phys. 11 (2008) 29.

[37] F. Sette, G. Ruocco, M. Krisch, U. Bergmann, C. Masciovecchio, V. Mazzacurati, G. Signorelli, R. Verbeni, Phys. Rev. Lett. 75 (1995) 850.

[38] U. Balucani, J.P. Brodholt, R. Vallauri, J Phys-Condens Mat 8 (1996) 6139.

[39] A. Cunsolo, M. Nardone, J. Chem Phys. 105 (1996) 3911.

[40] A. Cunsolo, G. Ruocco, F. Sette, C. Masciovecchio, A. Mermet, G. Monaco, M. Sampoli, R. Verbeni, Phys. Rev. Lett. 82 (1999) 775.

[41] G. Monaco, A. Cunsolo, G. Ruocco, F. Sette, Phys. Rev. E 60 (1999) 5505.

[42] R.D. Mountain, Rev Mod Phys 38 (1966) 205.

[43] A.I. Chumakov, G. Monaco, A. Monaco, W.A. Crichton, A. Bosak, R. Ruffer, A. Meyer, F. Kargl, L. Comez, D. Fioretto, H. Giefers, S. Roitsch, G. Wortmann, M.H. Manghnani, A. Hushur, Q. Williams, J. Balogh, K. Parlinski, P. Jochym, P. Piekarz, Physical review letters 106 (2011) 225501.

[44] W. Schirmacher, G. Diezemann, C. Ganter, Physical review letters 81 (1998) 136.

[45] R. Dell'Anna, G. Ruocco, M. Sampoli, G. Viliani, Physical review letters 80 (1998) 1236.

[46] U. Buchenau, M. Prager, N. Nücker, A.J. Dianoux, N. Ahmad, W.A. Phillips, Phys Rev B 34 (1986) 5665.

[47] J. Horbach, W. Kob, K. Binder, Eur Phys J B 19 (2001) 531.

[48] L.E. Bove, E. Fabiani, A. Fontana, F. Paoletti, C. Petrillo, O. Pilla, I.C.V. Bento, Europhys. Lett. 71 (2005) 563.

[49] L. Orsingher, G. Baldi, A. Fontana, L.E. Bove, T. Unruh, A. Orecchini, C. Petrillo, N. Violini, F. Sacchetti, Phys. Rev. B 82 (2010).

[50] T. Scopigno, E. Pontecorvo, R. Di Leonardo, M. Krisch, G. Monaco, G. Ruocco, B. Ruzicka, F. Sette, J. Phys.-Condens. Mat. 15 (2003) S1269.

[51] S. Hosokawa, W.C. Pilgrim, H. Sinn, E.E. Alp, Journal of physics. Condensed matter : an Institute of Physics journal 20 (2008) 114107.

[52] S. Hosokawa, M. Inui, Y. Kajihara, K. Matsuda, T. Ichitsubo, W.C. Pilgrim, H. Sinn, L.E. Gonzalez, D.J. Gonzalez, S. Tsutsui, A.Q. Baron, Physical review letters 102 (2009) 105502.

[53] V.M. Giordano, G. Monaco, Phys Rev B 84 (2011).

[54] X.G. Gong, G.L. Chiarotti, M. Parrinello, E. Tosatti, Europhys Lett 21 (1993) 469.

[55] V.M. Giordano, G. Monaco, Proceedings of the National Academy of Sciences of the United States of America 107 (2010) 21985.

[56] S. Hosokawa, S. Munejiri, M. Inui, Y. Kajihara, W.C. Pilgrim, Y. Ohmasa, S. Tsutsui, A.Q. Baron, F. Shimojo, K. Hoshino, Journal of physics. Condensed matter : an Institute of Physics journal 25 (2013) 112101.

[57] D.J. Thouless, Physics Reports 13 (1974) 93.

[58] M. Zanatta, F. Sacchetti, E. Guarini, A. Orecchini, A. Paciaroni, L. Sani, C. Petrillo, Physical review letters 114 (2015).

[59] Y.Q. Cai, D.S. Coburn, A. Cunsolo, J.W. Keister, M.G. Honnicke, X.R. Huang, C.N. Kodituwakku, Y. Stetsko, A. Suvorov, N. Hiraoka, K.D. Tsuei, H.C. Wille, J. Phys. Conf. Ser. 425 (2013) 202001. 
[60] C. Masciovecchio, U. Bergmann, M. Krisch, G. Ruocco, F. Sette, R. Verbeni, Nucl. Instrum. Meth. B

117 (1996) 339.

[61] M. Yabashi, K. Tamasaku, S. Kikuta, T. Ishikawa, Rev. Sci. Inst. 72 (2001) 4080.

[62] Y.V. Shvyd'ko, M. Lerche, U. Kuetgens, H.D. Ruter, A. Alatas, J. Zhao, Phys. Rev. Lett. 97 (2006) 235502.

[63] Y. Shvyd'ko, S. Stoupin, D. Shu, S.P. Collins, K. Mundboth, J. Sutter, M. Tolkiehn, Nature communications 5 (2014) 4219.

[64] A. Suvorov, D.S. Coburn, A. Cunsolo, J.W. Keister, M.H. Upton, Y.Q. Cai, Journal of synchrotron radiation 21 (2014) 473.

[65] A. Cunsolo, B.M. Leu, A.H. Said, Y.Q. Cai, The Journal of chemical physics 134 (2011) 184502.

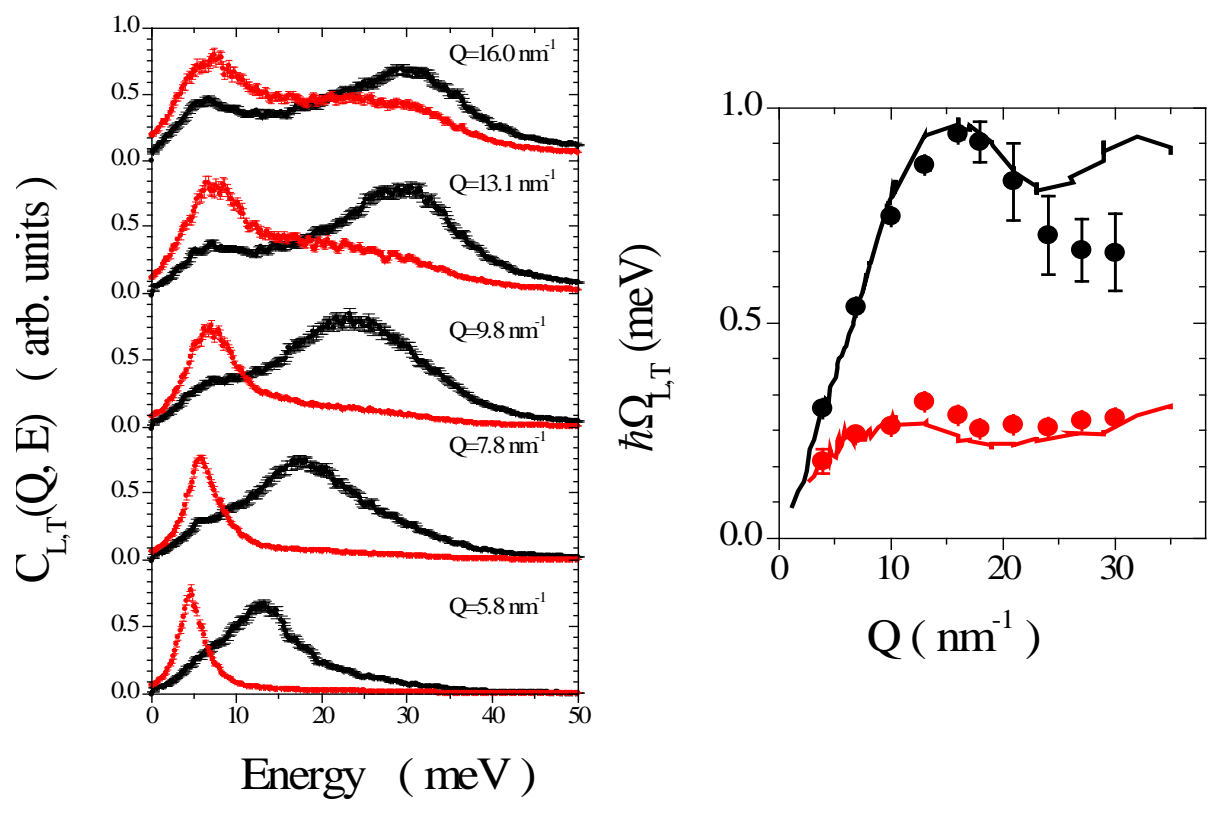

Figure 1: Left plot: MD simulation of the longitudinal (black dots) and transverse (red dots) current spectra are reported for same representative $Q$ values (data from Ref.[8]. In the left plot the dispersion curves of the longitudinal (black line) and transverse (red line) modes measured from the same work are compared with the positions of the high (black dots) and low frequency (red dots) peaks in the IXS spectra (from Ref. [2]). 


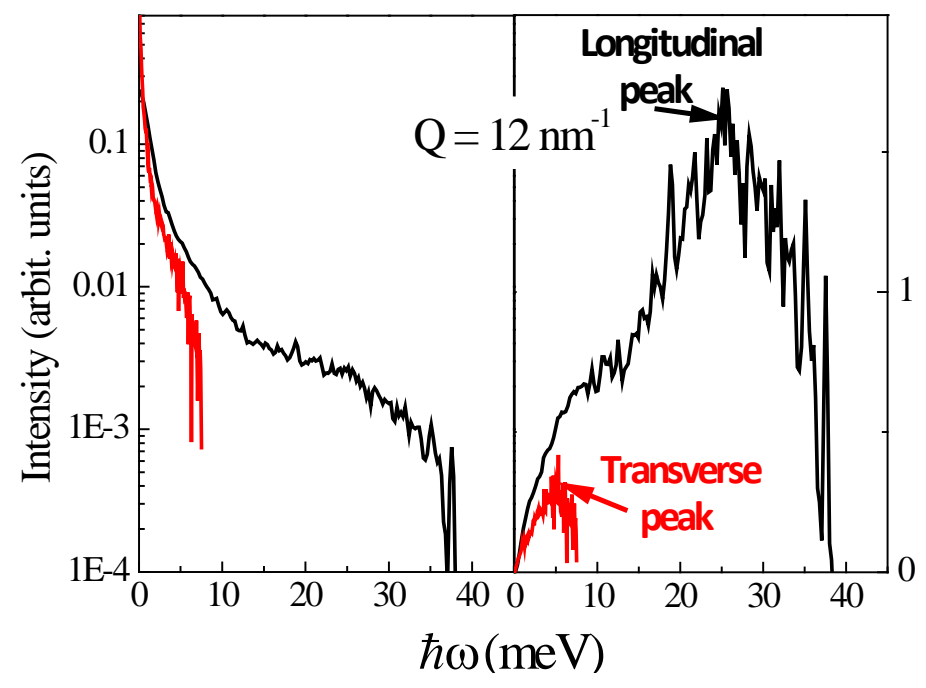

Figure 2: Left plot: IXS (red line) and INS (black line) spectral lineshape measured in Ref.[4]. Right plot: the corresponding current spectra are reported for reference. 


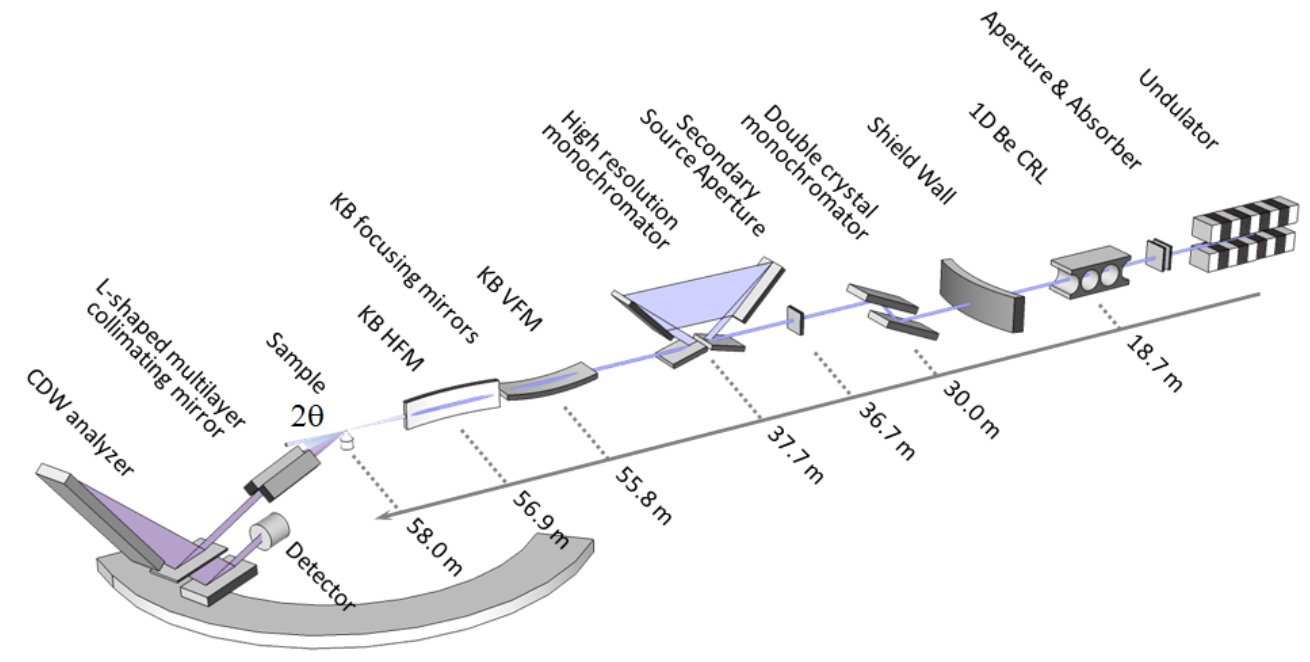

Figure 3: A schematic layout of the new high resolution IXS spectrometer soon available at NSLS II (from Ref. [64] ). 


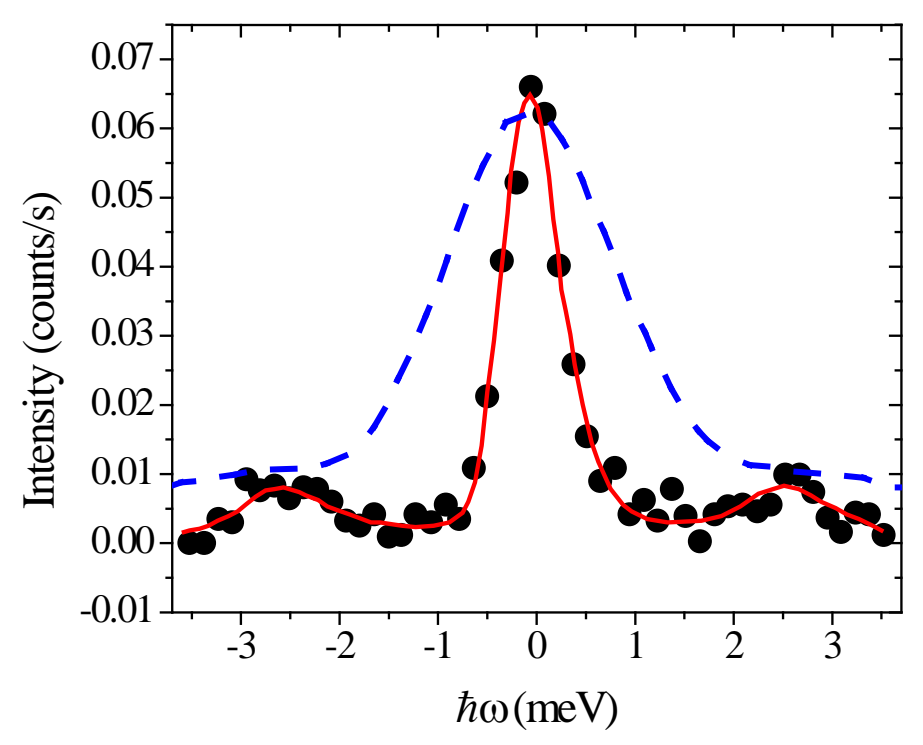

Figure 4: Extremely high resolution and spectral contrast IXS measurement of the spectrum of glycerol (dots) measured by a prototype of next generation IXS spectrometers (see text) and compared with the corresponding best fit lineshape (red line). The IXS spectrum of glycerol measured at $Q=1.5 \mathrm{~nm}^{-1}$ with state of art $1.5 \mathrm{meV}$ resolution spectrometer [65] is also reported for reference as a dashed line (all data are redrawn from Ref. [63]). 